\title{
Fiber-Optic Spectroscopy
}

National Cancer Institute

\section{Source}

National Cancer Institute. Fiber-Optic Spectroscopy. NCI Thesaurus. Code C116482.

The use of flexible, translucent fibers to measure the emission and absorption of different wavelengths of visible and non-visible light. 